\title{
Rapid Centroids and the Refined Position Accuracy of the Swift Gamma-ray Burst Catalogue
}

\author{
J.E. Hill ${ }^{\mathrm{a}, \mathrm{b}}$, L. Angelini ${ }^{\mathrm{a}, \mathrm{c}}$, A. Moretti ${ }^{\mathrm{d}}$, D.C. Morris ${ }^{\mathrm{e}}, \mathrm{J}$. Racusin ${ }^{\mathrm{e}}$, \\ D.N. Burrows ${ }^{\mathrm{e}}$, A.P. Beardmore, S. Campana ${ }^{\mathrm{d}}$, M. Capalbi ${ }^{\mathrm{g}}$, \\ J.A. Kennea ${ }^{\mathrm{e}}$, J.P. Osborne ${ }^{\mathrm{f}}$, C. Pagani, ${ }^{\mathrm{d}, \mathrm{e}}$, G. Tagliaferri ${ }^{\mathrm{d}}$, G. Chincarini ${ }^{\mathrm{d}}$, \\ N. Gehrels ${ }^{\mathrm{a}}$, A. Wells ${ }^{\mathrm{e}, \mathrm{f}}, \mathrm{J}$. A. Nousek ${ }^{\mathrm{e}}$ \\ ${ }^{a}$ NASA/Goddard Space Flight Center, Greenbelt, MD 20771, USA \\ ${ }^{b}$ Universities Space Research Association, Columbia, MD, 21044, USA \\ ${ }^{c}$ Department of Physics and Astronomy, The Johns Hopkins University, Baltimore, MD 21218, USA \\ ${ }^{d} I N A F$ - Osservatorio Astronomico di Brera, Merate, Italy \\ ${ }^{e}$ Department of Astronomy \& Astrophysics, Penn State University, USA \\ ${ }^{f}$ Physics \& Astronomy Department, University of Leicester, UK \\ ${ }^{g}$ ASI Science Data Center, 00044 Frascati, Italy
}

\begin{abstract}
The Swift X-ray Telescope autonomously refines the Burst Alert Telescope positions $\left(\sim 1-4^{\prime}\right.$ uncertainty) to better than 5 arcsec, within 5 seconds of target acquisition by the observatory for typical bursts. The results of the rapid positioning capability of the XRT are presented here for both known sources and newly discovered GRBs, demonstrating the ability to automatically utilise one of two integration times according to the burst brightness, and to correct the position for alignment offsets caused by the fast pointing performance and variable thermal environment of the satellite as measured by the Telescope Alignment Monitor. We present an evaluation of the position accuracy for both the onboard centroiding software and the ground software for the calibration targets and show that a significant improvement in position accuracy is obtained if the boresight detector position is optimised relative to the spacecraft pointing. Finally, we present an updated catalogue of Swift GRB X-ray positions obtained in Photon Counting Mode using the improved, calibrated boresight.

Keywords: Gamma-ray Bursts, X-rays, CCDs, Centroiding.
\end{abstract}

\section{INTRODUCTION}

The Swift X-ray position accuracy requirement is 5" within 5 seconds of the observatory settling on the GRB. This requirement applies to the initial observation of the GRB by XRT in Image mode prior to switching into an observation mode according to the source countrate (Hill et al. 2005a). When the source has faded to less than $\sim 1 \mathrm{mCrab}$, the XRT switches into Photon Counting (PC) mode. These data are analysed using the ground software to determine a refined position based on extensive follow-up and therefore better counting statistics. During the first 12 months on orbit, calibration and evaluation of the XRT position accuracy has provided improvements to the default parameters used for the position determination on-board and on the ground. This paper reports the position determination results to date. 


\section{Flight Software Position Accuracy}

Observations of X-ray calibration targets were performed in Image mode to calibrate and optimise the XRT Flight Software (FSW) centroiding algorithm. Analysis of these data led to updates to the onboard centroiding parameters, which were then verified with observations of additional fainter calibration targets. Figure 1 (left) shows the accuracy of the XRT onboard centroiding algorithm for a variety of source positions and brightness's. Figure 1 (right) shows how the centroiding accuracy depends on the source counts and integration time, and the flux at which the source is bright enough for the flight software to use a shorter integration time of $0.1 \mathrm{~s}$ rather than $2.5 \mathrm{~s}$. At the switch point between the long and short integration time, the total number of counts in the exposure is decreased and therefore the accuracy is decreased. As the flux is increased for the shorter exposure, the accuracy improves again.
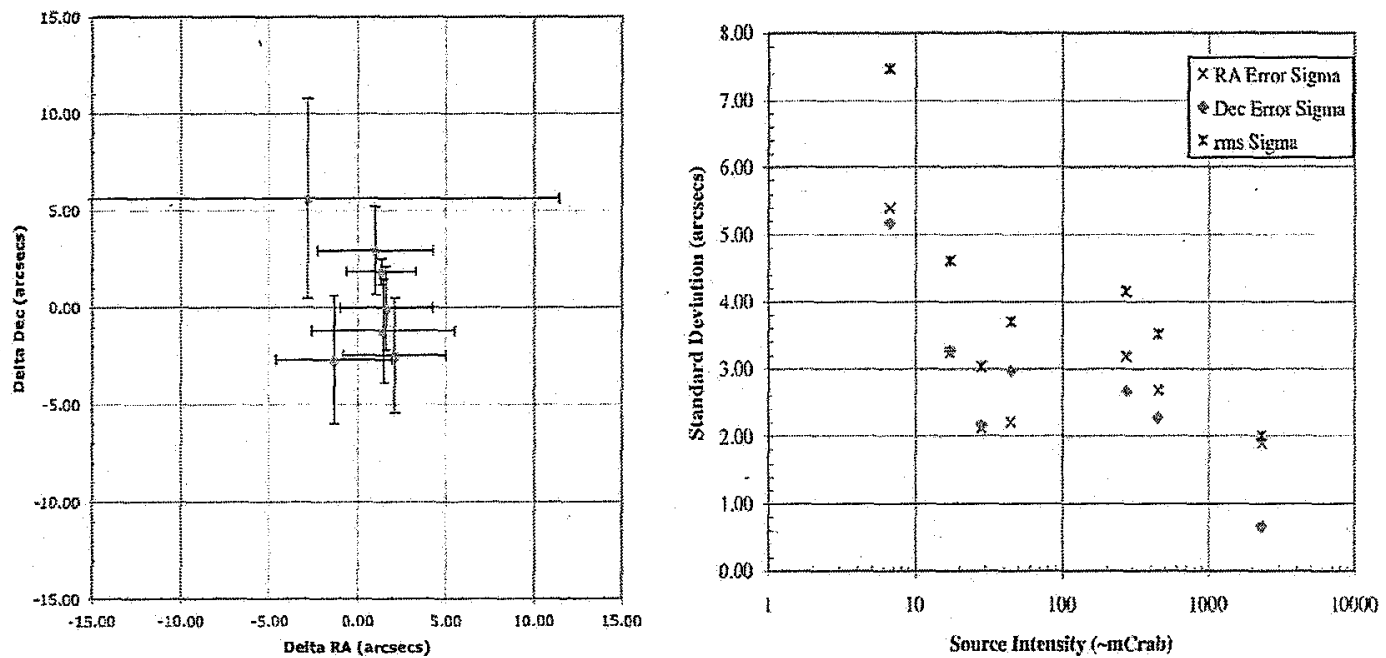

FIGURE 1. Left: XRT flight software position off-sets compared to the SIMBAD catalogue positions. Right: XRT flight software position off-sets versus source countrate, where $\sim 0.7 \mathrm{cnts} / \mathrm{sec} / \mathrm{mCrab}$

Between launch (20 November 2004) and 17 October 2005, the Burst Alert Telescope (BAT) has detected 84 bursts. 70 of the bursts were observed by the XRT, 68 of which were detected at $\mathrm{T}<200 \mathrm{ks}$. Four of the bursts were observed by the XRT during the prompt phase while the burst was still in progress (e.g. Hill et al. 2005b). The narrow field instruments followed up $70 \%$ of the bursts in less than 350 seconds after the BAT trigger and the XRT provided prompt positions $(<5 \mathrm{sec})$ in $41 \%$ of those cases. In the remaining cases the source was too faint or the XRT was in a calibration mode.

Following the initial boresight calibration between the XRT using Image mode and the Star Trackers, as an increasing number of roll angles were sampled, it became apparent that for some observations the positions obtained on the ground and onboard were less accurate and an update to the default boresight was necessary.

\section{Image Mode Boresight Analysis}

An idl script was written to recalculate source positions using different boresights, reproducing the FSW calculation. The script reads in the data from the header of the telemetered postage stamp message obtained in Image mode and regenerates the 
centroid position in RA and DEC with or without the Telescope Alignment Monitor (TAM) correction (Hill et al. 2005b). The script positions were verified against the onboard RA and DEC positions for the default boresight position $(300 \times 300)$ and found to be identical. The script was run for a matrix of boresight positions for each observation of 3 calibration sources. A position off-set was obtained for each observation by comparing the script position with the known optical position. An average off-set was calculated for each boresight position. From fitting a polynomial to the distribution of off-sets versus boresight position, a best-fit boresight position was obtained. This best-fit boresight was then verified against Image mode data of GRBs with optical counterparts. For the GRBs and the three calibration sources, the derived boresight was constant within the counting statistics. The largest variation from test-case to test-case was in the XRT $y$-axis. This analysis was performed for both TAM corrected and non-TAM corrected positions.

\section{Photon Counting Mode Boresight Analysis}

The best boresight position obtained for Image mode was verified against PC mode data where, due to the longer observing times, the counting statistics are, in general, much improved. 11 calibrations sources and 36 observations were selected with a distribution of roll angles. To limit the number of variables, this analysis was performed for data which were not corrected by the TAM.

Nine boresight positions were tested in a matrix around the best-fit boresight obtained from the Image mode data (e.g. Figure 2, 1eft). As in the Image mode analysis the average off-sets for each boresight position were plotted against the boresight $y$ and boresight $\mathrm{x}$-position. From fitting a curve to the data, the boresight was further refined to $298.2 \times 299.3$. This was confirmed as the best-fit position by reprocessing the observations with the refined boresight (Figure 2, right).
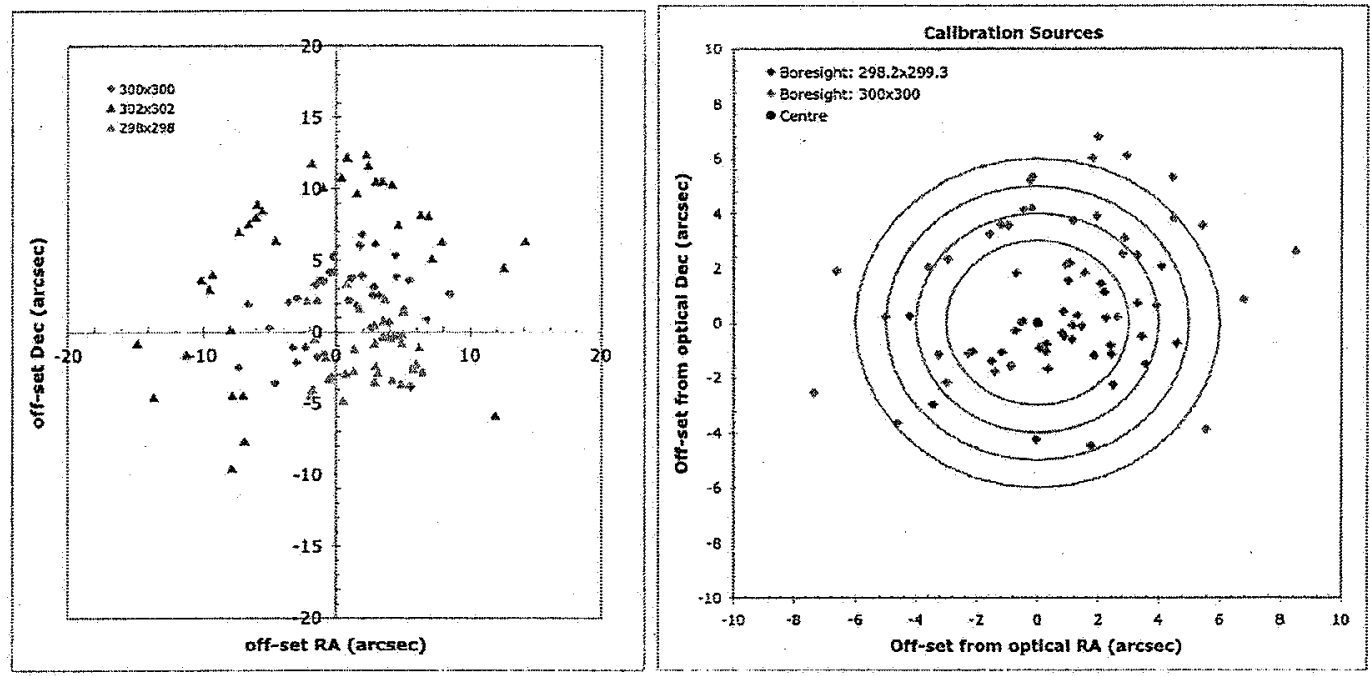

FIGURE 2. Left: Variation of position off-sets for 36 observations of 11 sources with three boresight positions. Default at $300 \times 300,298 \times 298$ and $302 \times 302$. Right: A comparison of calibration source position off-sets from optical positions for the default boresight and for the corrected boresight.

The mean off-set for the default boresight decreased from 4.5 " to 2.1 " for the new boresight. Additional verification was performed by plotting the off-set in RA and Dec against the roll angle of the observations and verifying that there was no accuracy 
dependence on roll angle. Following the boresight optimisation, an additional 12 sources were analysed over 35 observations with the new boresight. The average offset from the optical positions was found to be 2.2 " as seen in the previous sample, with $97 \%$ of the positions less than 5 " from the optical position.

\section{Gamma-ray Burst Refined Positions}

A catalogue of 37 GRBs with optical counterparts observed between December 2004 and October 2005 were analysed with the default boresight (Figure 3, left) and the best-fit boresight position of $298.2 \times 299.3$ (Figure 3, right). The average off-set from the optical counterpart was 2.3 ", the same as that obtained for the calibration sources. A significant improvement in accuracy can be seen in Figure 3. All 64 GRBs detected by XRT between December and October have been reprocessed with the new boresight and the updated positions are within the $90 \%$ error circle of the positions in Moretti et al. 2005.
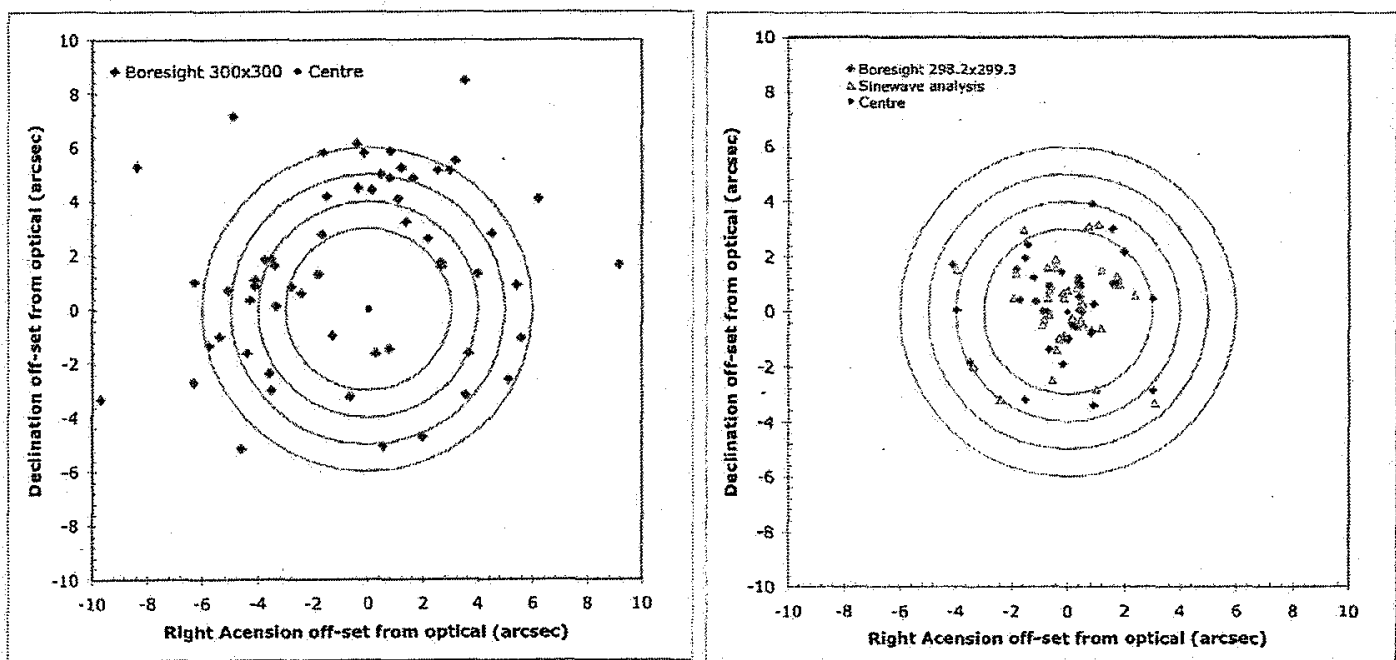

FIGURE 3. Left: XRT ground processed GRB positions for the default $300 \times 300$ boresight. Right: A GRB position off-sets from optical positions for a sinewave corrected fit and using a corrected boresight.

\section{ACKNOWLEDGEMENTS}

This work is supported at Penn State by NASA contract NAS5-00136; at the University of Leicester by the Particle Physics and Astronomy Research Council on grant number PPA/Z/S/2003/00507; and at $\mathrm{OAB}$ by funding from ASI on grant number $\mathrm{I} / \mathrm{R} / 039 / 04$.

\section{REFERENCES}

Hill, J.E. et al., "The unique operating Modes of the Swift X-ray Telescope”, 2005, Proc. SPIE, 5898, 589815-1

Hill, J.E, et al., The Astrophysical Journal, 2006, in press

Moretti et al, Astronomy \& Astrophysics, 2005, submitted 


\section{Rapid Centroids and the Refined Position Accuracy of the Swift Gamma-ray Burst Catalogue}

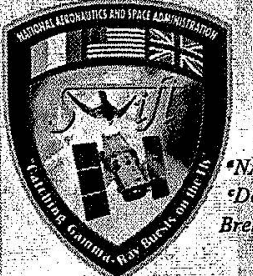

J.E Hill, b. L. Angelinia,c, A. Moretti, D.C. Montse I. Racusine, D.N. Burrows, A.P.

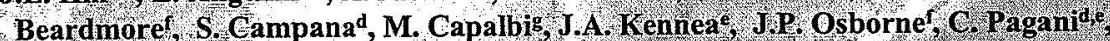
G. Tagliaferrid, G. Chincarini, N Gehrels, A. Wellser, J.A. Nouseke

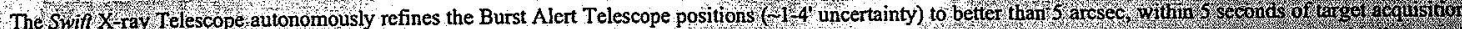

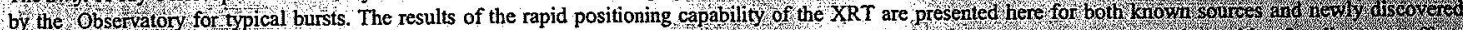

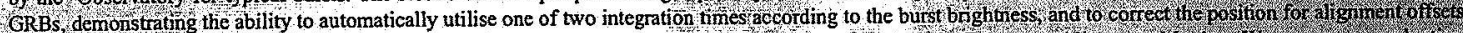

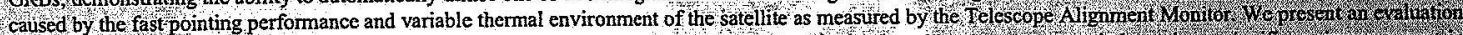

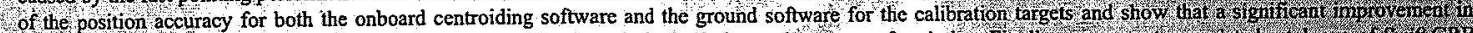
7. X ray positions obtained in Photon Counting Mode using the improved, calibrated boresight

\section{Flight Software Positions}

Observations of calibration targets were performed in Image Mode on DOY 32,33 and 34 to calibratc and optimise the XRT FSW centroiding algorithm. A salysis of these data led to updates to the onboard centroiding parameters, which were then verified with observations of additional fainter calibration targets obtained on DOY 60. Figure 1 shows the accuracy of the XRT onboard centroiding algorithm for:a variety of source positions and brightness's.
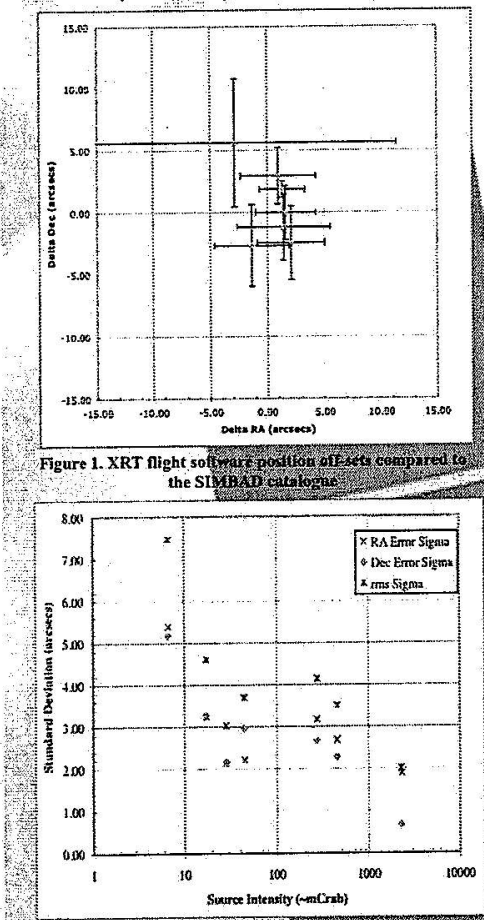

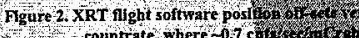

Figure 2 shows how the depends on the source coun and where the source is bight software to use the shorter inte sec At the switch point between integration time, for a $01 \mathrm{I} \mathrm{sec}$ number of total number of coun therefore the accuracy is decrease becomes bright enough again. Betwcen the launch of Swift and I the BAT observed 84 birsts 70 of 2 2. observed by the XRT, 68 of which Were detected at $T<200$ ks. Four of the bursts were observed by the XRT during the prompt phase, $70 \%$ (49/70) of the bursts were followed up in less than 350 seconds and XRT provided prompt positions ( $\angle 5$ sec) in 20 cases The XRT GRB positions compared to the optical counterpart positions between I Aprl (end of the commissioning phase) and 3 August are shown in Figure 3. The FSW positions incorporatc a correction as measured by the Telescope Alignment Monitor, the Best X ray positions are those obtained from ground processing nultiple orbits of data but do not include the TAM corrcction.

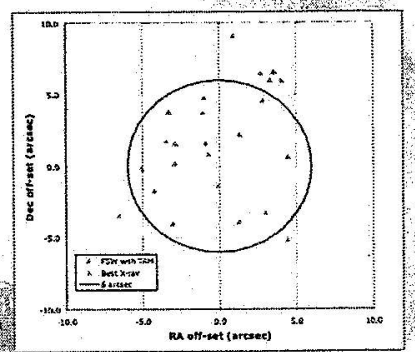

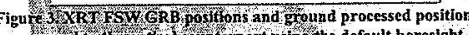
From the Image Mode dat obtantined of calibration sourcks d best th to boresight position was derived in $\mathrm{CCD}$

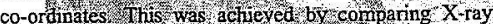
source positions with the optral position oyer many

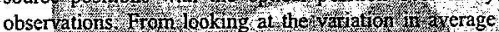
observations

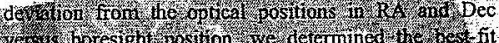

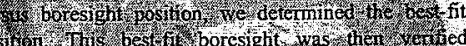

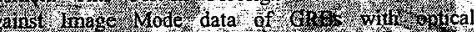

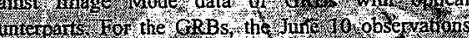

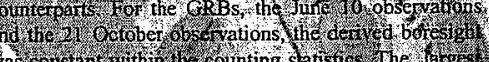

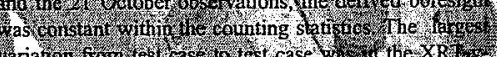

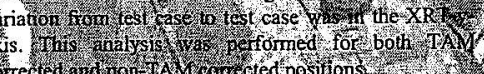

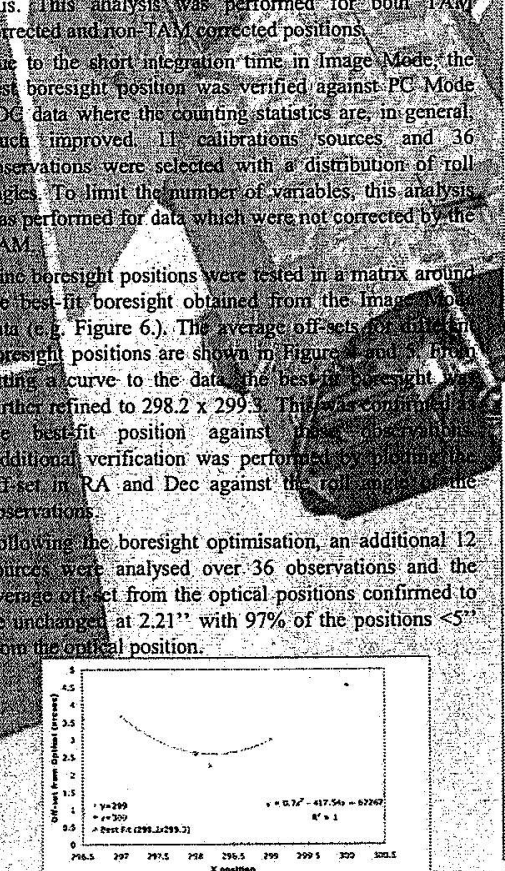
Figure 4 Best fit boresight in pixcls, XRT X-axis

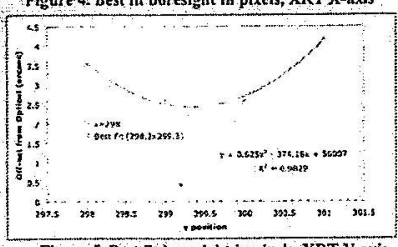

Figure 5. Best fit boresight in pixels, XRT Y-axis

\section{Boresight Corrected Positions}
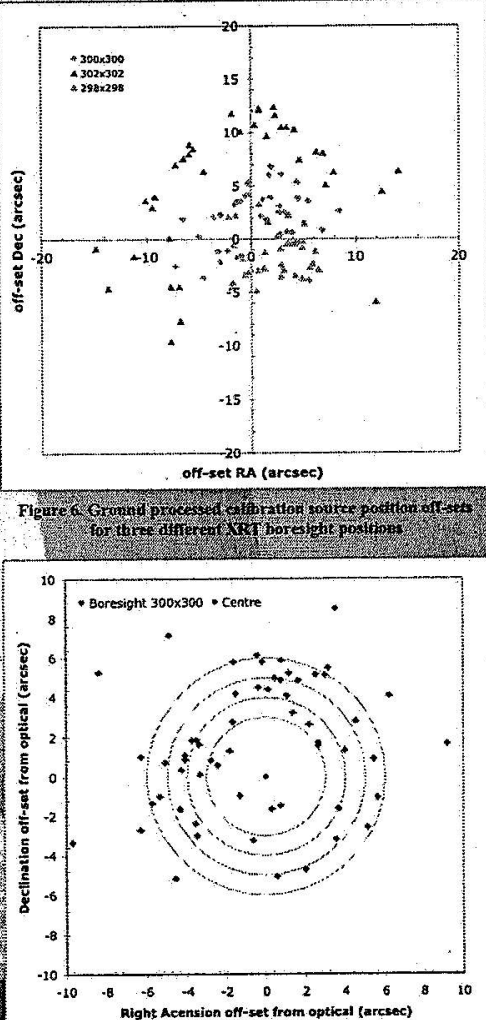

7 7. J

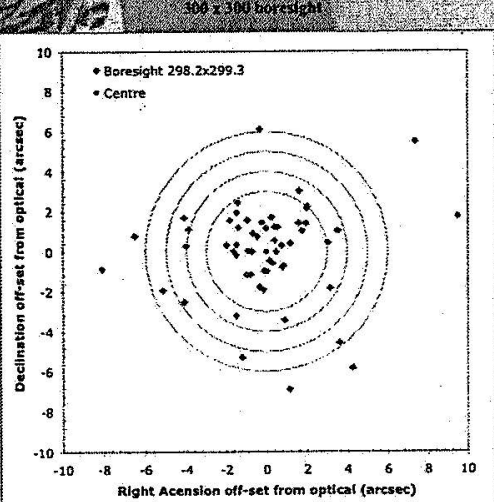

Flgure 8. Borestight corrected GRB positions: $298.2 \times 299.3$

A catalogue of 64 GRBs observed between December and October were analysed with the best fit boresight position of $298.2 \times 299.3$. The average distance from the optical counterparts was $2.3 \%$, equivalent to that obtained for the calibration sources. The off-sets for all $64 \mathrm{GRB}$ pasitions with the default and the corrected boresight are shown in Figures 7 and 8 , respectively. A marked improvement in accuracy can be seen. 